\title{
BMJ Open Challenges and facilitators for health professionals providing primary healthcare for refugees and asylum seekers in high-income countries: a systematic review and thematic synthesis of qualitative research
}

\author{
Luke Robertshaw, ${ }^{1}$ Surindar Dhesi, ${ }^{2}$ Laura L Jones ${ }^{1}$
}

To cite: Robertshaw L, Dhesi S, Jones LL. Challenges and facilitators for health professionals providing primary healthcare for refugees and asylum seekers in high-income countries: a systematic review and thematic synthesis of qualitative research. BMJ Open 2017;7:e015981. doi:10.1136/ bmjopen-2017-015981

- Prepublication history and additional material are available. To view these files, please visit the journal online (http://dx.doi. org/10.1136/bmjopen-2017015981).

Received 19 January 2017 Revised 30 March 2017 Accepted 18 May 2017

\section{(a) CrossMark}

${ }^{1}$ Institute for Applied Health Research, University of Birmingham, Birmingham, UK ${ }^{2}$ Department of Earth and Environmental Sciences, School of Geography, University of Birmingham, Birmingham, UK

Correspondence to

Dr. Laura L Jones;

L.L.Jones@bham.ac.uk

\section{ABSTRACT}

Objectives To thematically synthesise primary qualitative studies that explore challenges and facilitators for health professionals providing primary healthcare for refugees and asylum seekers in high-income countries.

Design Systematic review and qualitative thematic synthesis.

Methods Searches of MEDLINE, EMBASE, PsycINFO, CINAHL and Web of Science. Search terms were combined for qualitative research, primary healthcare professionals, refugees and asylum seekers, and were supplemented by searches of reference lists and citations. Study selection was conducted by two researchers using prespecified selection criteria. Data extraction and quality assessment using the Critical Appraisal Skills Programme tool was conducted by the first author. A thematic synthesis was undertaken to develop descriptive themes and analytical constructs.

Results Twenty-six articles reporting on 21 studies and involving 357 participants were included. Eleven descriptive themes were interpreted, embedded within three analytical constructs: healthcare encounter (trusting relationship, communication, cultural understanding, health and social conditions, time); healthcare system (training and guidance, professional support, connecting with other services, organisation, resources and capacity); asylum and resettlement. Challenges and facilitators were described within these themes.

Conclusions A range of challenges and facilitators have been identified for health professionals providing primary healthcare for refugees and asylum seekers that are experienced in the dimensions of the healthcare encounter, the healthcare system and wider asylum and resettlement situation. Comprehensive understanding of these challenges and facilitators is important to shape policy, improve the quality of services and provide more equitable health services for this vulnerable group.

\section{INTRODUCTION}

Throughout human history, countless people have been forced to flee from their homes
Strengths and limitations of this study

- This is the first review to systematically identify and synthesise qualitative research exploring challenges and facilitators for health professionals providing primary healthcare for refugees and asylum seekers.

- Thematic synthesis of studies from a range of countries and primary healthcare settings allows identification of common, generalisable themes with potential to influence policy and practice.

- The review was limited to English language studies, which may have led to over-representation of studies conducted in English-speaking high-income countries.

- The review was limited to core, clinical health professionals: doctors nurses and midwives.

and countries due to violence or threats of violence. Other nations may provide refuge for those seeking a safe haven, and in 1950, the Office of the United Nations High Commissioner for Refugees (UNHCR) was established to provide international leadership and coordination for the protection of refugees and promotion of their well-being. ${ }^{1}$ The UNHCR convention defines refugees as persons who have a 'well-founded fear of being persecuted for reasons of race, religion, nationality, membership of a particular social group or political opinion, is outside the country of his nationality and is unable or, owing to such fear, is unwilling to avail himself of the protection of that country; or who, not having a nationality and being outside the country of his former habitual residence as a result of such events, is unable or, owing to such fear, is unwilling to return to it'. ${ }^{2}$ Those in the application process to be granted refugee status are referred to 
as 'asylum seekers'. By the end of 2015, there were an estimated 65.3 million forcibly displaced people worldwide, including 40.8 million internally displaced people, 21.3 million refugees and 3.2 million asylum seekers. ${ }^{3}$

Refugees and asylum seekers are a vulnerable group with significant and complex health needs. ${ }^{4}$ A survey by the UK Border Agency in 2010 showed refugees to be in poorer health than the general population. ${ }^{5}$ As most refugees and asylum seekers originate from low-income and middle-income countries, there are, accordingly, higher prevalences of pre-existing infectious diseases such as hepatitis B, tuberculosis and HIV compared with host populations. ${ }^{6}$ The risk of contracting infectious diseases may be increased by poor hygiene conditions during flight from conflict, coupled with insufficient vaccine coverage. $^{7}$ Studies have also highlighted the sexual and reproductive health needs of this group, ${ }^{8}$ with high levels of sexual gender-based violence (SGBV) being reported along with limited access to contraception. ${ }^{8}{ }^{9}$ Refugees and asylum seekers also suffer from non-communicable diseases such as hypertension, musculoskeletal disease, chronic respiratory disease and diabetes, which may be undermanaged and exacerbated when they are forced to flee their countries. ${ }^{10}$

A further concern for refugee and asylum seeker populations is their mental health. Violence experienced in countries of origin, including war, sexual abuse and torture are reported, which may lead to psychological and physical trauma. ${ }^{11}$ These premigration traumas are compounded by postmigration stressors such as loss of social networks, shifting societal roles and cross-cultural stress while integrating into countries of settlement. ${ }^{12}$ Fazel $e t a l^{13}$ estimated that $9 \%$ of adult refugees may suffer with post-traumatic stress disorder, which is approximately 10 times estimates in an age-matched American population. $^{13}$

Primary healthcare teams are on the frontline of healthcare provision for refugees and asylum seekers that arrive in high-income countries. ${ }^{14}$ These teams may include a variety of professional backgrounds, clinical and non-clinical, but typically include a core of general practitioners, community-based nurses and midwives. ${ }^{15} 16$ These health professionals face significant challenges when caring for refugees and asylum seekers. ${ }^{17-19}$ They must address their complex health and social needs, often in cross-cultural interactions, and operate within health systems that may not be structurally configured or politically favourable towards this group. ${ }^{17-20}$ These challenges impact on their ability to provide the same quality of care as the general population, leading to healthcare inequalities. ${ }^{20} 21$

Experiences of health professionals caring for refugees and asylum seekers in high-income countries have been investigated through a range of qualitative research studies conducted across several countries and primary healthcare settings. A recent systematic review by Suphanchaimat $e t a l^{22}$ synthesised challenges providing healthcare services to migrants from a provider perspective. The review included a minority of studies that had refugees and asylum seekers as service users, focused purely on challenges of healthcare provision, and adopted a limited, purposive search strategy. To our knowledge, this present review is the first to synthesise experiences of health provision for migrants defined specifically as refugees and asylum seekers; synthesise both challenges and facilitators for health professionals and adopt a systematic approach to identification of qualitative research. Therefore, this review aims to systematically identify and thematically synthesise challenges and facilitators experienced by health professionals that provide primary healthcare for refugees and asylum seekers in high-income countries.

\section{METHODS}

This systematic review sought qualitative research studies as they are the appropriate design for understanding perceptions and experiences of healthcare provision. ${ }^{23}{ }^{24}$ Systematic identification and synthesis of these studies may consolidate the current evidence-base, increase the breadth and depth of understanding and provide more generalisable conclusions than individual primary studies. ${ }^{25} 26$

This review was guided by established methodology for systematic review and thematic synthesis of qualitative research, outlined by Thomas and Harden. ${ }^{27}$ Thematic synthesis of data, applied in this methodology, is suited to development of recommendations for practice and policy and provides a transparent link between conclusions and the primary studies synthesised. ${ }^{27} 28$ Reporting of this review has been guided by Enhancing Transparency of Reporting the Synthesis of Qualitative Research (ENTREQ) framework. ${ }^{29}$

\section{Search strategy}

The following databases were searched from inception until week 3 of March 2016: MEDLINE, EMBASE, PsycINFO, CINAHL and Web of Science. The search strategy was based on the Sample, Phenomenon of interest, Design, Evaluation, Research type (SPIDER) tool, which has been developed as an alternative to Population, Intervention, Comparison, Outcome (PICO) to optimise identification of qualitative studies for evidence syntheses. ${ }^{30}$ Search terms were combined for primary health professionals/healthcare, refugees and asylum seekers and qualitative research. No language or date limits were applied. The full detailed search strategy is documented in online supplementary file 1. Further hand-searches were conducted based on included studies' reference lists and citations (in Google Scholar).

After removal of duplicates, titles and abstracts were screened by one researcher (LR), excluding articles that clearly did not meet the inclusion criteria. Full-texts of remaining articles were obtained and assessed by two independent researchers, according to prespecified study selection criteria (detailed below). Disagreements were resolved via discussion. 


\section{Box 1 Definitions of challenge and facilitator}

Challenge: a factor that inhibits, obstructs or creates difficulties for health professionals when providing primary healthcare. Facilitator: a factor that promotes, enables or assists health professionals when providing primary healthcare.

\section{Selection criteria}

This review included peer-reviewed, qualitative primary research studies that met the following criteria: English language; explored challenges or facilitators (defined in box 1) for health professionals providing primary healthcare to refugees and asylum seekers (including forced migrants, involuntary migrants or refugee claimants); and were conducted in a high-income country as defined by the World Bank country classification $2015 .{ }^{31}$ Studies were limited to those from high-income countries because of the authors' interest in developing recommendations for policy and practice applicable to advanced primary healthcare systems.

Mixed methods studies were included if the qualitative element's methods and results could be isolated for synthesis. As definitions of health professionals in primary healthcare teams are diverse ${ }^{16}$ this review was limited to articles that interviewed core clinical healthcare professionals including: general practitioners, nurses, pharmacists and midwives working in primary healthcare settings. Articles were excluded if they were not based on peer-reviewed primary qualitative studies (ie, reviews, case studies, reports, opinion pieces) or were conducted in a secondary care setting. Articles that had referred to service users as 'migrants' or 'immigrants' were excluded, as these terms have a broader meaning including economic migrants, students and family unification. ${ }^{32}$ Those that referred to 'illegal immigrants' or 'undocumented migrants' were also excluded as they are known to have unique characteristics (eg, ineligible for free healthcare) that would not be typical of refugees and asylum seekers. ${ }^{33}$ Articles interviewing mental health professionals were excluded as this clinical area has specific characteristics. Studies that contained a mixture of eligible and ineligible participants were only included if data for eligible participants could be isolated for synthesis. Studies were also excluded if the full-text articles could not be obtained through institutional access or from requests sent to authors through Research Gate. The full inclusion and exclusion criteria applied in this review are documented in online supplementary file 2 .

\section{Data extraction}

Study characteristics were extracted by one author (LR) using a data extraction proforma. Characteristics included aims, setting, participants, methodology, results and recommendations/applications. Findings (results) and discussion sections from included articles were imported into NVivo V.11 software (NVivo qualitative data analysis Software; QSR International, V.11, 2016) for analysis.

\section{Assessment of quality}

Included articles were assessed by one author (LR) using the Critical Appraisal Skills Programme (CASP) tool for appraisal of qualitative research. ${ }^{34}$ Articles were not excluded from the synthesis or given weighting based on this assessment, as there is currently no accepted method for this in syntheses of qualitative research. ${ }^{35}$ All articles were included irrespective of their reporting quality given that they contributed to the conceptual richness of the synthesis. Where articles used mixed methods, only the qualitative element was appraised.

\section{Data synthesis}

A thematic synthesis was conducted broadly following the methodology outlined by Thomas and Harden. ${ }^{27} \mathrm{An}$ article, considered data-rich (containing numerous challenges and facilitators), was selected as an index-article and uploaded into NVivo V.11 software. The findings (results) and discussion sections were coded inductively within the two categories of 'challenges' and 'facilitators', as defined by the review question. This approach of inductive coding within a priori categories follows established methodology seen in similar qualitative syntheses. ${ }^{36}$ Primary quotations, author's commentary and author's interpretations were coded. Sections were only coded if they contained challenges or facilitators (box 1), and referred to the health professionals defined for this review. Following the index-article, subsequent articles were coded using the same method in approximate order of descending data-richness. Concepts in each article were coded to iteratively develop and refine a codebook, with each article having an ability to contribute new codes. Once all articles had been coded, the finalised codebook was applied across all articles. The final codebook was analysed to inform descriptive themes closely resembling the prevailing concepts across primary studies. These themes were discussed and agreed within the research team. An analytical model was then developed to create higher-order constructs within which descriptive themes were located.

\section{RESULTS}

\section{Systematic search and selection}

Systematic database searches identified 5970 articles. A further 16 articles were identified through handsearching of reference lists and citations. After removal of duplicates, 3571 articles remained. A total of 3493 articles were excluded based on the title and abstract. Full-texts of the remaining 78 articles were sought for detailed assessment against the inclusion criteria. Nine of these articles could not be obtained. In addition, due to resource limitations, four non-English language studies were unable to be translated and assessed against the selection criteria. After reviewing the 65 available full-text papers and applying the full selection criteria, 26 articles were included in the thematic synthesis (figure 1). 


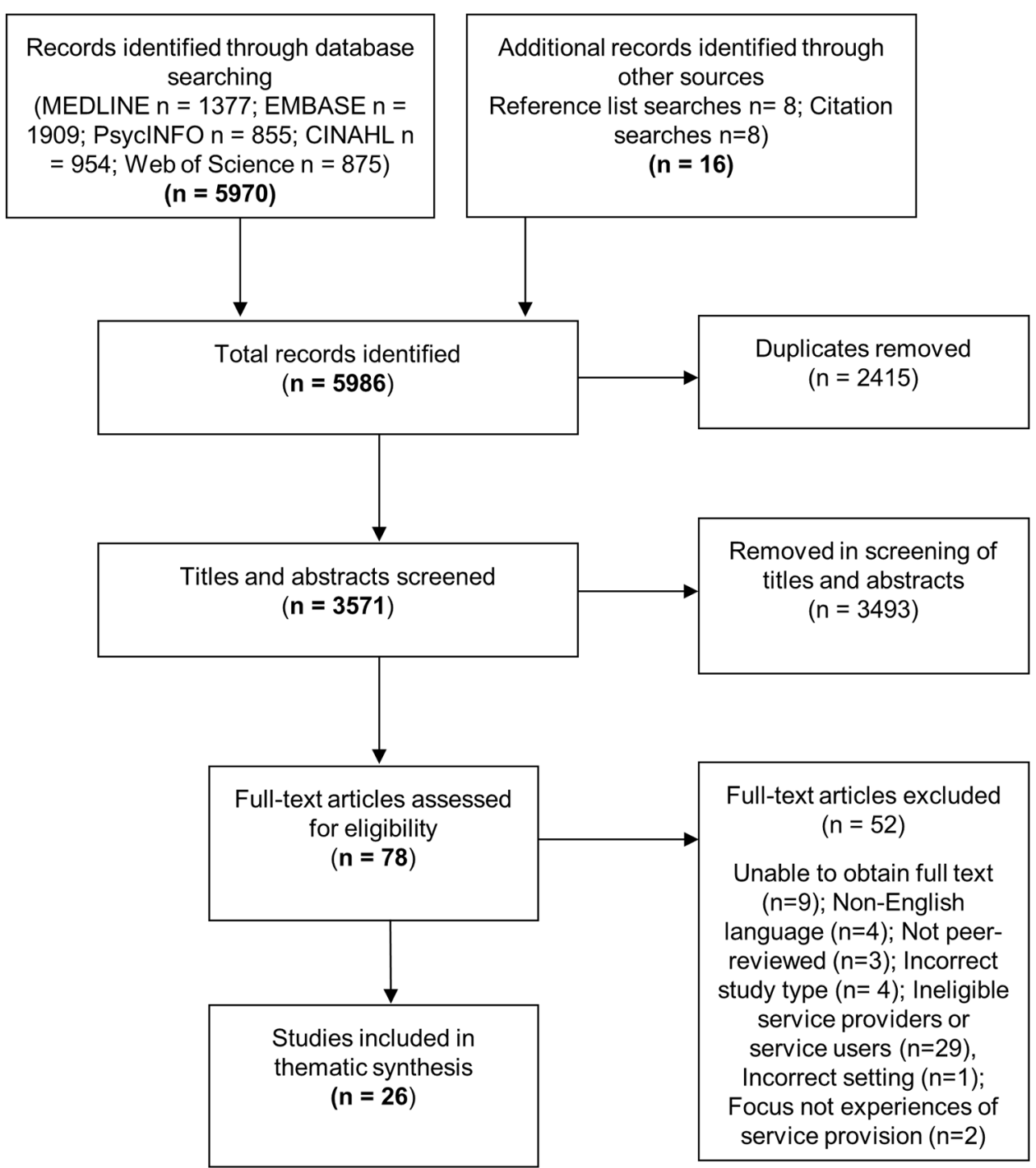

Figure 1 Flow diagram of systematic search and study selection.

\section{Characteristics of included studies}

The 26 articles included were based on 21 primary studies, of which 19 were qualitative studies ${ }^{17} 18$ 37-58 and 2 were mixed methods. ${ }^{19}$ Nine articles were from Australia, ${ }^{41} 4345-48505758$ seven from the UK, ${ }^{18} 37-404259$ three from the Netherlands ${ }^{452} 53$ and one from each of Denmark, ${ }^{17}$ Switzerland, ${ }^{19}$ New Zealand, ${ }^{49}$ Sweden,${ }^{51}$ the USA, ${ }^{54}$ Ireland ${ }^{55}$ and Canada. ${ }^{56}$ All articles were published between 1999 and 2016. Service users were described as 'refugees' in 11 articles, ${ }^{174} 43-495456$ 'asylum seekers' in 6 articles, ${ }^{1}{ }^{3852535559}$ 'refugees and asylum seekers' in 5 articles, ${ }^{1837} 394042$ 'of refugee background' in 3 articles ${ }^{50} 5758$ and 'involuntary migrants' in 1 article. ${ }^{51}$

Qualitative data extracted for this synthesis were derived from 357 participants with a combined sample of 194 nurses, 35 midwives and 128 doctors. None included pharmacists. Data collection methods varied across the 21 primary studies represented, with 14 solely using individual interviews (including in-depth, semi-structured, unstructured), ${ }^{17-19}$ 37-40 42 44 45 47-4951555659 one employed group interviews only ${ }^{54}$ and four combined individual and group interviews. ${ }^{4346505758}$ One study used observational methods and individual interviews, ${ }^{41}$ and one combined group interviews and qualitative questionnaires. ${ }^{52}{ }^{53}$ Table 1 summarises characteristics of included articles and online supplementary file 3 contains the complete data extraction.

\section{Quality assessment}

Application of the CASP critical appraisal tool revealed variable results across the 26 articles assessed. All except one article $^{39}$ gave a clear statement of the research aims. The majority (21 articles) ${ }^{17-19}$ 37-40 42-44 464849 51-58 sufficiently described the sampling strategy and provided some rationale for participants' selection. Possible reasons for non-participation were discussed in only four articles. ${ }^{19} 374647$ The data collection method was stated in all articles, however, the extent of information provided about interview schedule's content was variable. A significant number did not describe the setting of data collection (13 articles) $3843-4750525356-59$ or the identities of interviewers (12 articles). ${ }^{18} 1938-424647555859$ Only eight 


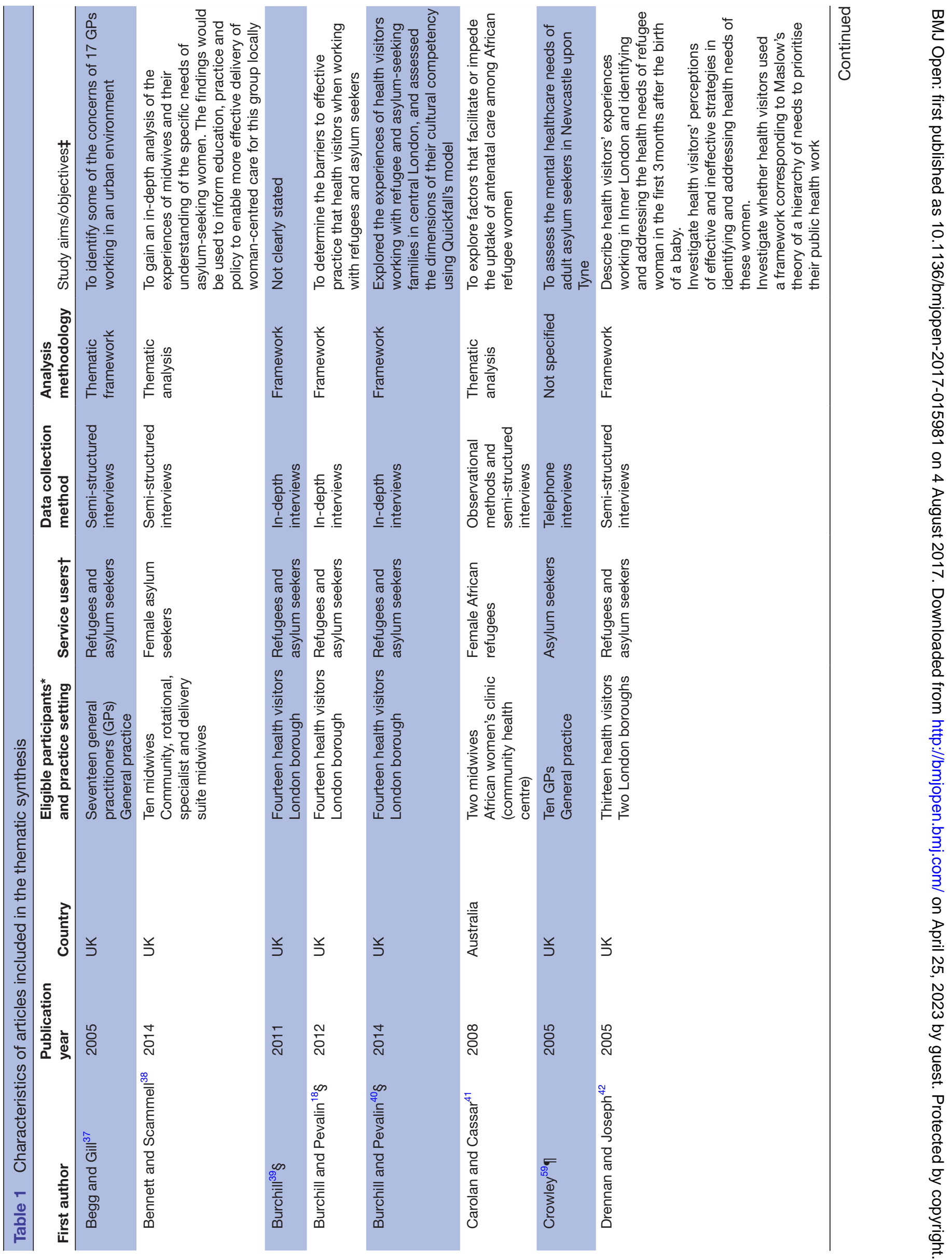




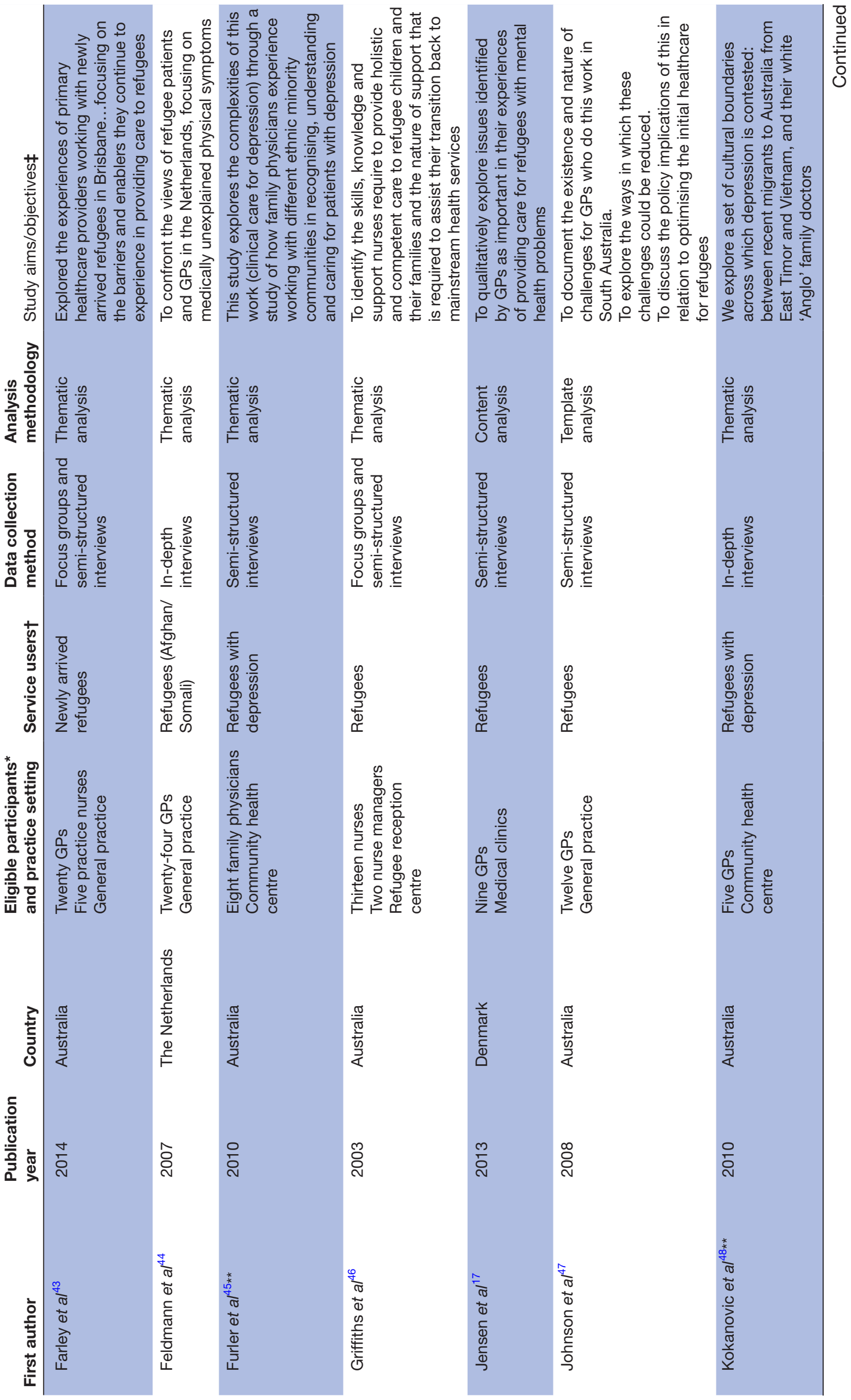




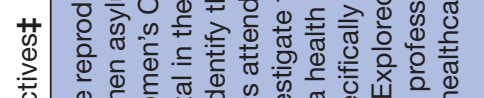

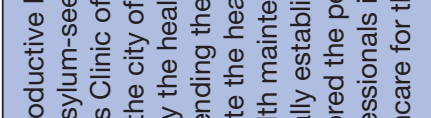

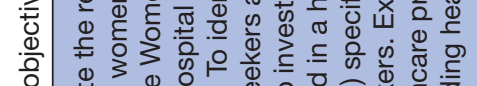

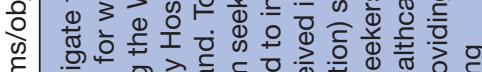

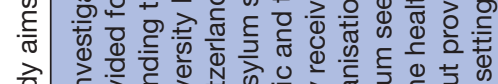

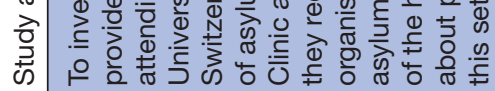

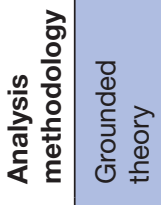

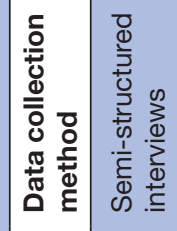

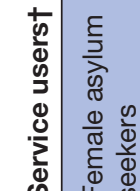

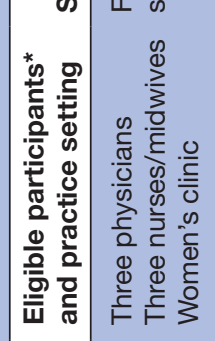

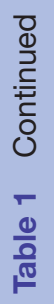

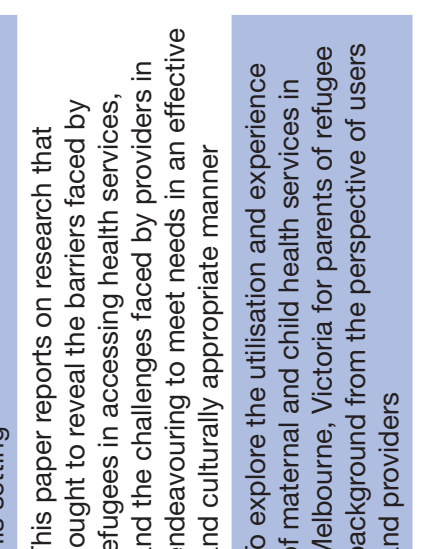

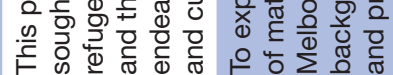

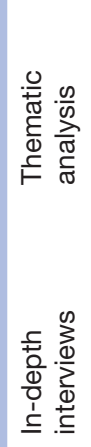

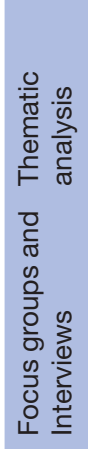

0
$\mathbb{0}$
$\mathbb{0}$
$\stackrel{9}{0}$
$\mathbb{0}$
$\simeq$

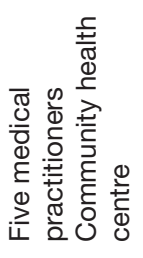

$\frac{0}{5}$
$\frac{\pi}{\pi}$
$\stackrel{0}{0}$
$N$
$\frac{1}{0}$
$\frac{0}{2}$

¿̊ำ

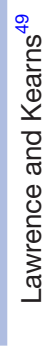

$+$
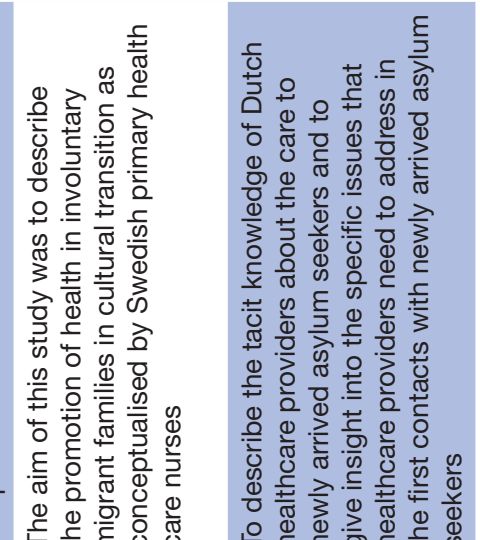

तี

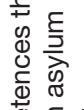

要荡

हो.

뜰

责部市

要

은 훙

응

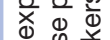

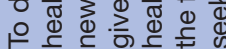

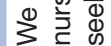

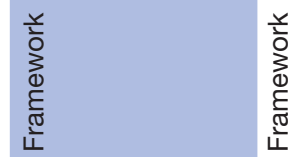

$\stackrel{\infty}{\stackrel{0}{*}}$

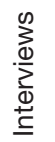

离

离

ㅇํㅇ

을 을 을

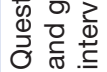

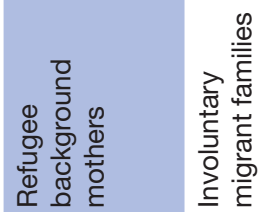

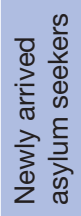

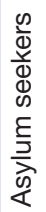

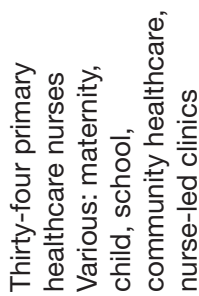

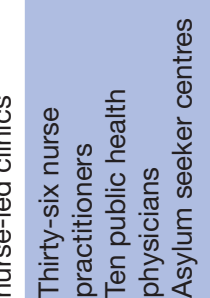

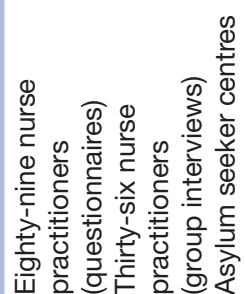




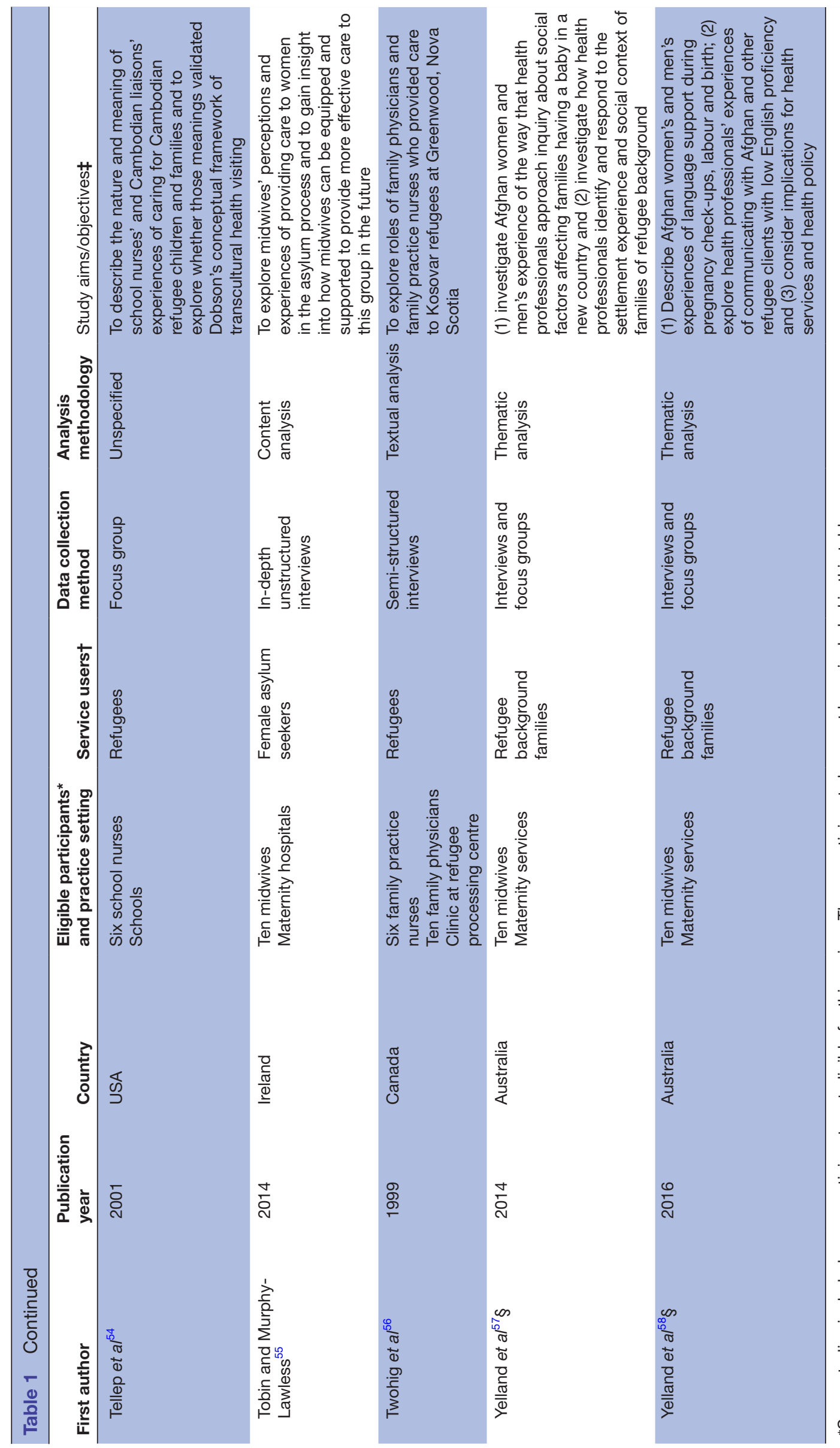

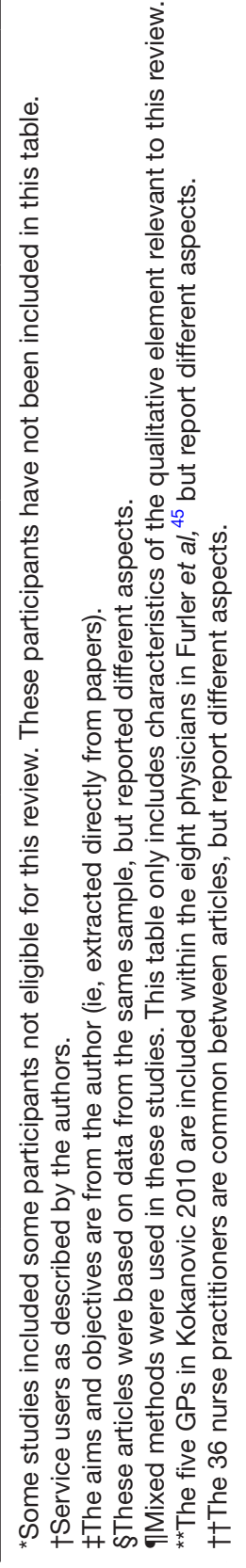


articles $^{43}$ 47-50 525659 gave justification for chosen data collection methods or interview settings. Data saturation was rarely discussed, featuring in five articles. ${ }^{37} 43474856$

Reflexivity was particularly poorly discussed across articles. Only seven 37394348515455 reflected on potential bias and influence of researchers at any stage in the study (formulation of review question, sampling, data collection or analysis).

Ethical approval was described in the majority of articles (23 articles), ${ }^{17-19}$ 37-43 45-48 50-58 but they often lacked sufficient information to judge whether ethical standards had been followed. Thirteen articles $^{17-19} 38-4348515355$ described how participants were informed about the nature and purpose of the study, 17 articles $^{17} 19373840424348-5660$ described obtaining consent and 12 articles $^{173741-4346} 4751-55$ discussed how confidentiality was assured or maintained.

The approach to data analysis was described to some extent in all but one article ${ }^{59}$; however, there was variation in the level of detail given. Involvement of multiple researchers in the analysis process was reported in 19 articles. ${ }^{17-19} 37$ 39-43 45-48 50 51 55-58 The majority (25 articles) ${ }^{17-1937-58}$ gave support for findings with references to primary data (eg, quotations from participants). Findings were generally clearly presented and discussed in context of wider research literature, policy and practice, although a few (six articles) ${ }^{39} 4042495456$ were limited in this area. Ten articles ${ }^{19} 3741434550-5357$ explicitly reflected on the credibility of their findings.

Full details of the CASP assessment are provided in online supplementary file 4 .

\section{Thematic synthesis findings}

Challenges and facilitators for health professionals providing primary healthcare to refugees and asylum seekers were interpreted within 11 descriptive themes, embedded in 3 analytical constructs: healthcare encounter (trusting relationship, communication, cultural understanding, health and social conditions, time), healthcare system (training and guidance, professional support, connecting with other services, organisation, resourcing and capacity) and asylum and resettlement. Figure 2 illustrates the relationships between analytical constructs and descriptive themes. Healthcare encounters occur within the environment of healthcare systems, both of which operate within wider asylum and resettlement policies and processes. Table 2 provides a taxonomy of challenges and facilitators and table 3 contains illustrative quotations from primary studies for each descriptive theme.

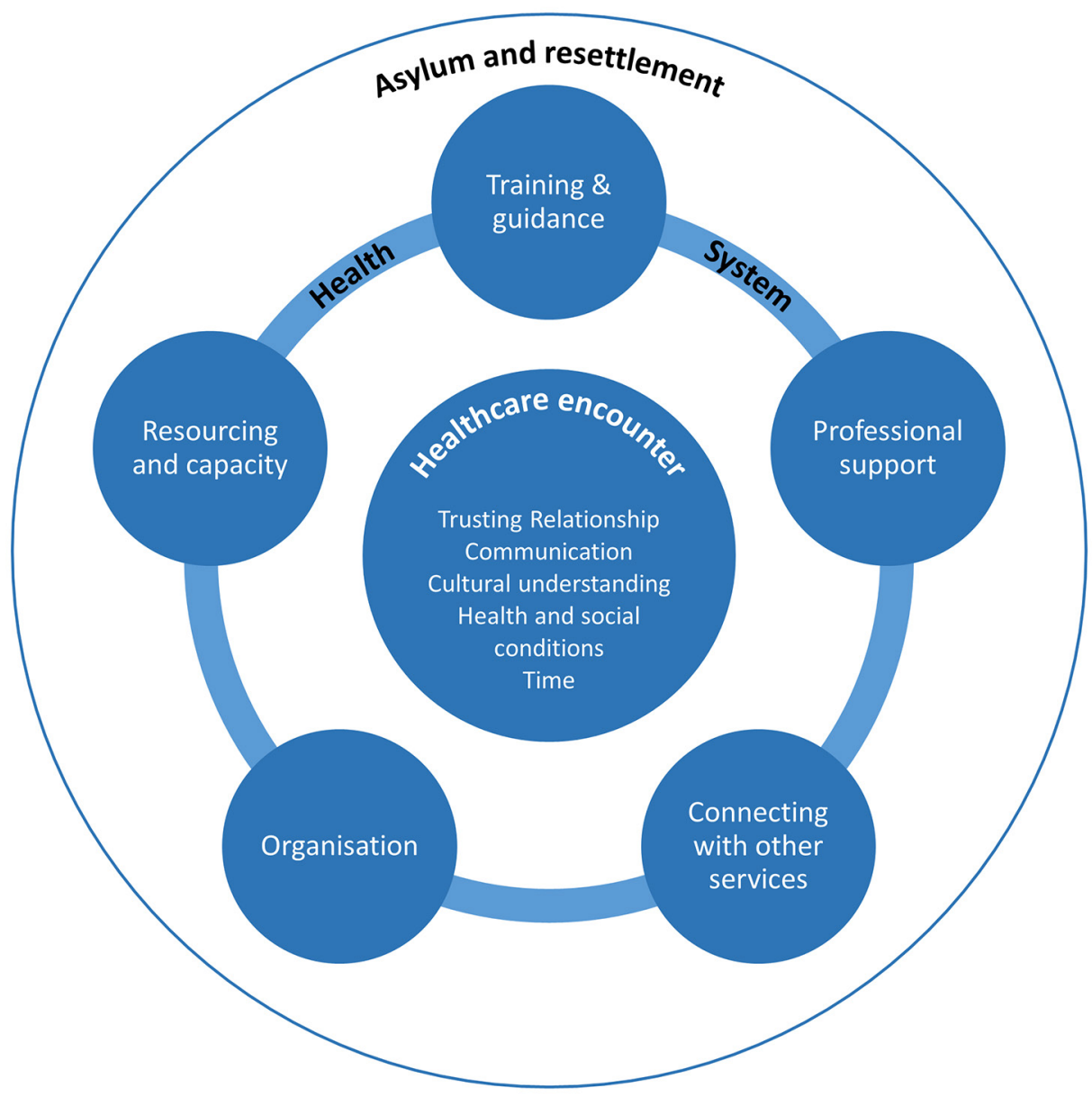

Figure 2 Model illustrating analytical constructs and descriptive themes. 
Table 2 Taxonomy of challenges and facilitators

\begin{tabular}{lll}
\hline $\begin{array}{l}\text { Analytical } \\
\text { construct }\end{array}$ & Descriptive theme & Challenges \\
\hline Healthcare & Trusting & -Transience of refugees/ asylum seekers \\
encounter & relationship & -Suspicion of authorities
\end{tabular}

Trusting

relationship
-Suspicion of authorities

\section{Facilitators}

-Continuity of care

-Assisting with wider needs

-Taking an interest

-Compassion/empathy

-Explaining role

Communication -Language: assessing case history/gaining consent/ ensuring patient understanding

-Interpreters: additional time/expense, unavailability, inaccuracy/imposition of own views

-Telephone interpreters: impersonal, technological

failures

-Illiteracy

-Lack of language specific resources

Cultural

understanding

-Different understandings of health concepts/

terminology/healthcare systems

-Understanding patient's symptoms

-High expectations of patients

-Different cultural values

Health and social -Physical: communicable diseases, female genital conditions mutilation, injuries

-Unusual diseases

-Psychological: torture, abuse, social difficulties, somatisation

-Lacking skills, knowledge, support

Time

-Increased time requirement

-Increased duration/occurrences of appointments

-Insufficient time: rushed appointments

-Time taken away from other patient groups

The healthcare Training and

system guidance

-Lack of training/guidance

-Lack of awareness of available resources

-Time constraints

Professional support

-Deficiency of professional support

-Supporting traumatised patients without support -Isolation

Connecting with -Referral difficulties; services not present/not suitable other services

-Difficulty understanding/navigating healthcare system

Organisation

Resourcing and -Increased costs

capacity -Funding shortages

-Workforce shortages

-Inflexibility/unsuitability of interpreter services

$\begin{array}{lll}\text { Asylum and } & \text { Asylum and } & \text {-Policy restrictions } \\ \text { resettlement } & \text { resettlement } & \text {-Conflicts of interest } \\ & & \text {-Understanding changing policy environment and } \\ & \text { healthcare provisions } \\ & & \text {-Perceived abuses of system }\end{array}$

-Visual aides
-Interpreters: professionally trained, continuity

-Telephone interpreters: increased availability

-Knowledge of other cultures: values, health practices, body language

-Personal qualities: sensitivity, empathy, cultural humility

-Training

-Guidance

-Professional support

-History taking 
Table 3 Illustrative quotations

\begin{tabular}{ll} 
Theme & Quotation and reference* \\
\hline Healthcare encounter &
\end{tabular}

Healthcare encounter

Trusting Challenge "... you put your mind around trying to sort things out, the dreadful things that have happened relationship to them, and then the next week it will be a different family there and you start the whole process all over again, trying to build up some sort of trust..."42

Facilitator "Creating trust is an important aspect, to show that you are interested in the person, not only
in the disease; to show that you want to know something about the context. Sometimes it is
difficult to find time for it in a busy practice, but I see it is a worthwhile investment". ${ }^{4}$

Facilitator "Everything comes down to communication. To know what's going on, what they need, what you need, because it's a partnership, isn't it?"18

'... this (telephone interpreting) is available 24 hours and is instantaneous... it is revolutionised, all the doctors use it, the receptionists, the nurses...

Cultural understanding
Challenge

'...they have a different culture, so their cultural perception of symptoms and what they mean... trying to interpret the difference between a bloated abdomen and a painful abdomen, just becomes an impossible task. ${ }^{43}$

"I sometimes say, 'I am only a doctor'. Sometimes there are far greater expectations than what you can honour"17

"I think most people understand sadness... but in some cultures, they don't understand (depression) as a condition that requires treatment ${ }^{1145}$

Facilitator '...there were specialised nurses who had worked overseas, who gave workshops for us, and explained much of the history, and explained some of the conflicts which they bring over here'. 54

Health and social Challenge conditions

"I am quite overwhelmed at times as to how complex these ladies' lives are... "38
"I guess it is out of our comfort zone, because our medical experience doesn't include the exotic illnesses that they front up with..."47

"Midwives spoke of the emotional impact of working with women with trauma histories: 'How does it affect me, you just feel sad you know, but you just do the best that you can and that's all you can do"

Facilitator "(Specialist team teaching sessions) is the sort of thing that people need to help give them a baseline of knowledge, and I suppose, the support to realise that there are other people they can talk to, to help them and signpost, or help them to signpost their clients in the right direction". 40

'We do not need to know the whole lot; we do not need the whole case history [...] to have a bit more understanding'. ${ }^{38}$

Time Challenge ‘... generally speaking a consultation with a refugee will take twice as long (as with) a local patient'. ${ }^{37}$

'...providing care with interpreters was more time consuming than without, meaning that midwives had to "juggle their time" to facilitate good care'. ${ }^{38}$

\section{Healthcare system}

Organisation Facilitator

'The flexibility of the general practice setting enabled providers to act on their commitment to provide refugee healthcare, allowing them to be responsive and innovative in their approach to caring for refugees and also providing flexibility in the hours they work'. ${ }^{43}$

'Participants felt that significant gains had been made to the refugee healthcare system, with the establishment of a specialised service. One provider working in the field for some time described thinking... fantastic, finally'43

$\begin{aligned} & \text { Resourcing and Challenge } \\ & \text { capacity }\end{aligned}$
$\begin{aligned} & \text { somebody else (this) needs to be acknowledged'. }{ }^{37} \\ & \text { "But I was more angry that I just needed more hands to help. So, for me it was about practical } \\ & \text { support". } 46\end{aligned}$


Table 3 Continued

\begin{tabular}{|c|c|c|}
\hline Theme & & Quotation and reference* \\
\hline $\begin{array}{l}\text { Training and } \\
\text { guidance }\end{array}$ & Challenge & $\begin{array}{l}\text { "Even when we called... the [Division of General Practice]... they didn't know how to guide } \\
\text { us... I think we didn't have a guideline..." } 43\end{array}$ \\
\hline \multirow[t]{2}{*}{$\begin{array}{l}\text { Professional } \\
\text { support }\end{array}$} & Challenge & $\begin{array}{l}\text { '...lack of institutional support all contributed to varying feelings of powerlessness on the part } \\
\text { of the midwives themselves'. } 55\end{array}$ \\
\hline & Facilitator & $\begin{array}{l}\text { 'They described the value of currently available external supports, including language classes, } \\
\text { translation and interpreting services and specialised refugee health services, particularly in the } \\
\text { area of mental health'. }{ }^{3}\end{array}$ \\
\hline $\begin{array}{l}\text { Connecting with } \\
\text { other services }\end{array}$ & Challenge & $\begin{array}{l}\text { "She explained she had seen a lot of problems...I put her touch with a voluntary (nationality } \\
\text { specific) counselling organization to then discover she had to pay and she can't afford it". }{ }^{2}\end{array}$ \\
\hline \multicolumn{3}{|c|}{ Asylum and resettlement } \\
\hline & Challenge & $\begin{array}{l}\text { 'These requirements differed: on the one hand to be the caregiver, to be the patient's advocate } \\
\text { in fact, and on the other to act as advocate of the Federal Office for Refugees, and thirdly to } \\
\text { be responsible for the organisation, to save costs for the health insurance. But that is simply } \\
\text { not possible'. }{ }^{19}\end{array}$ \\
\hline & & $\begin{array}{l}\text { "I don't know if there is some sort of system that they go through, or some sort of protocol } \\
\text { that they, medically, have to go through before they are granted visas... "47 }\end{array}$ \\
\hline
\end{tabular}

*Participant's quotations are in italics, study authors text is normal typeface.

\section{The healthcare encounter}

Challenges and facilitators for healthcare provision to refugees and asylum seekers were experienced within the healthcare encounter. This is the milieu of personal engagement between health professionals and service users. Five inter-related factors influenced health professionals' practice: trusting relationship, communication, cultural understanding, health and social conditions and time.

\section{Trusting relationship}

Buildingtrustingrelationshipswithrefugeesorasylumseekers featured in 15 of the articles. ${ }^{1840-42} 44-464850-5557$ Facilitators included continuity of the attending care provider 4250525457 ; taking an active interest in their background, language and culture ${ }^{40445457}$ and assisting them with their wider needs. ${ }^{18} 4050$ Having a compassionate and empathetic disposition was also seen as important in relationship building. ${ }^{4151525557}$ The transient nature of some service users made building relationships challenging ${ }^{42}$ and trust was threatened when refugees or asylum seekers thought that healthcare professionals were associated with immigration authorities. ${ }^{38} 4252$ Health professionals found that clearly explaining their role and confidentiality brought reassurance and allayed suspicions. ${ }^{42}{ }^{52}$ Some benefits of establishing trusting relationships were said to be increased engagement with the healthcare service by refugees and asylum seekers, ${ }^{18} 404150$ and greater levels of disclosure about their health and social concerns. ${ }^{42} 48505257$

\section{Communication}

Communication was a theme found in 22 included articles. $^{17-19} 373840$ 42-45 47-58 The language barrier was widely cited as challenging while caring for refugees and asylum seekers. ${ }^{17-19} 3738404344$ 47-51 53-58 Individual articles elaborated that language barriers presented difficulties in assessing case histories, ${ }^{19}$ gaining consent ${ }^{55}$ and ensuring patients understood treatment. ${ }^{42}$

Using interpreters was considered a major facilitator in communication ${ }^{173738404345505157}$ and was maximised when interpreters were well-trained and familiar with medical terminology. ${ }^{1745}$ Continuity of the interpreter was deemed important in fostering good communication and increased confidence in the integrity of translation. ${ }^{38404550}$ There were, however, challenges associated with interpreter use. ${ }^{17-1937384042434547495052555658}$ Communicating through interpreters required additional time ${ }^{3847}$ and financial expense. ${ }^{55}$ Suitable interpreters were not always available at the appropriate time, ${ }^{17} 3842434755$ which could lead to delayed, extended or rearranged appointments. ${ }^{173847}$ This led, in some cases, to family or other community members being asked to translate instead of professional interpreters. ${ }^{42}$ Participants were also concerned that interpreters did not always accurately communicate $^{37} 4043455556$ and may impose their own views. ${ }^{40}$ The use of telephone interpreters received mixed opinions. Advocates welcomed the increased availability of interpreters at any time of the day, ${ }^{37}$ but others 
felt they were more impersonal ${ }^{5058}$ and pointed to technological failures that hindered communication. ${ }^{50} 58$

Further communication challenges included unavailability of written health information in service users' language ${ }^{5357}$ and in some cases patients were unable to read or write. ${ }^{43}$ To improve communication with those with limited language skills, some participants used objects or other visual aids. ${ }^{51}$

\section{Cultural understanding}

Cultural understanding was a theme described across 21 articles. ${ }^{17} 18373840-4951-57$ Healthcare provision could be challenging, when there were different understandings of health, illness or healthcare. ${ }^{17} 184044-49515355$ Health literacy could be limited ${ }^{43} 4753$ and different terms could be used to refer to health conditions. ${ }^{18454857}$ Healthcare concepts such as preventative care (eg, screening), ${ }^{47} 49$ mental healthcare ${ }^{4857}$ and self-management ${ }^{51}$ were sometimes unfamiliar. Service users also lacked understanding of host countries' healthcare systems, ${ }^{374042434549}$ making them prone to miss appointments, ${ }^{43}$ and attempt to inappropriately access services. ${ }^{37}$

Differences in health culture presented difficulties for health professionals' understanding of patient's symptoms ${ }^{45}$ and required additional time and effort explaining health conditions, healthcare concepts or health systems. ${ }^{42} 4751$ It was also reported that some refugees or asylum seekers had very high, and sometimes unrealistic, expectations of health services or health professionals, ${ }^{1737405253}$ which needed to be counteracted by participants. ${ }^{1753}$ Disparities in cultural values such as gender roles, decision-making, social taboos and time-orientation were also mentioned as challenges, ${ }^{41} 474853$ with some health professionals expressing uncertainty about approaching some clinical tasks such as physical examinations. ${ }^{47}$

Gaining knowledge and understanding about cultures of refugees and asylum seekers was viewed as an important facilitator in cross-cultural care. ${ }^{38} 40424752545557$ This included understanding differences in values, ${ }^{42}$ body language ${ }^{52}$ health practices ${ }^{42}$ and health presentations. ${ }^{52}$ Cultural understanding allowed health professionals to adjust their healthcare practice accordingly. ${ }^{40} 454849515556$ Personal qualities in health professionals that were deemed to enhance cross-cultural interactions were sensitivity, ${ }^{4254}$ empathy ${ }^{40454}$ and cultural humility. ${ }^{5455}$

\section{Health and social conditions}

Health professionals spoke of challenges in dealing with physical, psychological and social problems that were typically presented by refugees and asylum seekers. ${ }^{173740} 43444647525355-57$

Physical conditions presented challenges 3740434447 and included tropical diseases such as malaria and schistosomiasis ${ }^{43}$; other communicable diseases such as TB and HIV ${ }^{37} 4044$; and nutritional deficiencies. ${ }^{37} 4044$ Physical injuries were also encountered, such as female genital mutilation $(\mathrm{FGM})^{40} 55$ and injuries inflicted from conflict or torture. ${ }^{40}$ Health professionals did not always feel prepared or equipped to deal with these conditions $^{43} 47$ and there were concerns from general practitioners that some conditions could remain undiagnosed. ${ }^{434} 47$

Psychological conditions were considered challenging to deal with, ${ }^{1737404346525355-57}$ and were frequently seen among refugees and asylum seekers. ${ }^{3743}$ These included psychological trauma related to war, ${ }^{17}$ torture ${ }^{4043} 46$ and other abuses. ${ }^{17} 3840$ Postmigration stresses were also perceived to impact negatively on their mental health such as the asylum and resettlement process ${ }^{174047}$ social isolation $^{174555}$ and other social vulnerabilities. ${ }^{40} 5057$ Health professionals found engaging with these service users emotionally difficult ${ }^{37} 55$ and distressing when hearing their disturbing stories. ${ }^{40424655}$ They also expressed feelings of powerlessness ${ }^{174655}$ believing they lacked required skills, knowledge and support to respond to their complex psychological needs. ${ }^{43} 57$

A further challenge noted by health professionals across four articles was the manifestation of medically unexplained symptoms (somatisation) among some refugees and asylum seekers, ${ }^{18} 434448$ which could be frustrating ${ }^{43}$ and time consuming to address. ${ }^{43} 48$

Several facilitators were identified that could help deal with complex physical and psychological conditions. Careful history-taking of medical, social and migration background was helpful ${ }^{3844505357}$ and could identify possible risk factors. ${ }^{53} 57$ Training in conditions common among refugees and asylum seekers was deemed valuable, ${ }^{37384046525355}$ increasing confidence in care delivery ${ }^{40}$ and resulting in 'more effective, evidencebased care' ${ }^{38}$ Clinical guidelines for refugee healthcare were considered beneficial, ${ }^{37} 47$ although these were often unavailable. ${ }^{37}{ }^{47}$ Professional support was regarded as a facilitator, ${ }^{37} 384243465155$ provided within services ${ }^{42}$ or from external organisations specialising in refugee healthcare. ${ }^{43} 46$ The importance of psychological support for those working with traumatised patients was highlighted, ${ }^{465155}$ such as counselling or debriefing. ${ }^{46} 51$ Challenges around training, guidance and professional support are described in 'The healthcare system' section.

\section{Time}

A significant challenge faced by health professionals was the time required to provide healthcare for refugees and asylum seekers. ${ }^{1837} 3840434749-51555659$ More time was necessary due to the aforementioned challenges around building relationships, ${ }^{18} 3840$ communication, ${ }^{38} 505559$ achieving cultural understanding ${ }^{47}$ and dealing with complex health conditions. ${ }^{18} 38475051$ This additional time demand meant that appointments needed to be extended in duration ${ }^{3747}$ or occur more frequently. ${ }^{18} 49$ Health professionals were concerned that time limitations could lead to 'rushed consultations ${ }^{59}$ and the potential to miss some conditions. ${ }^{59}$ Some also commented that the extra time spent caring for refugees and asylum seekers drew them away from other patient groups. ${ }^{40} 43$ 


\section{The healthcare system}

Health systems have been defined as the combination of resources, organisation, financing and management that culminate in the delivery of health services to the population' ${ }^{61}$ They are the environment in which healthcare encounters take place. Healthcare professionals described health system-related challenges and facilitators within five areas: training and guidance, professional support, connecting with other services, organisation and resourcing and capacity.

\section{Training and guidance}

As already described in 'health and social conditions', health professionals thought that specific training and guidance would facilitate their clinical practice, improving their competence and confidence. Positive examples of training delivered were: orientation to services and resources available for refugees and asylum seekers ${ }^{40}$; culture-specific information ${ }^{42}{ }^{54}$; engaging with women about $\mathrm{FGM}^{40}$ and trauma-sensitive care. ${ }^{46}$ Despite this, a broad base of participants identified lack of training, education or guidance as detrimental to practice. ${ }^{173738424346495055}$ Even when available, training may be inaccessible due to lack of awareness or time constraints. ${ }^{43}$ Participants called for more training, guidance or information regarding integration with other health and social care services, ${ }^{37} 4250$ health profiles of specific groups, ${ }^{46}$ cultural awareness/competence ${ }^{42} 46474950$ and the wider process of asylum. ${ }^{37} 42$

\section{Professional support}

As reported in the earlier section 'Health and social conditions', professional support was needed by health professionals working with refugees and asylum seekers. However, professional support was identified as deficient in healthcare systems. ${ }^{37} 434655$ Participants in one study described "isolation" 43 that they felt within the healthcare system and another study described support networks as 'non-existent' ${ }^{37}$ Concerns were raised that health professionals exposed to distressing stories were not provided with sufficient psychological support. ${ }^{465}$

\section{Connecting with other services}

Connecting with other health and social care services was another important facilitator for health professionals. ${ }^{17} 183840424749-5254$ Establishing referral pathways to different services in the healthcare system ${ }^{40} 42475152$ and services within civil society ${ }^{4042} 47$ could direct refugees and asylum seekers to appropriate care. Some health visitors described accompanying refugees and asylum seekers to support groups to help with introductions. ${ }^{40}$ Good communication and cooperation between services was helpful ${ }^{38}$ and fruitful collaborations with other services were recognised, such as delivering services together ${ }^{5051}$ and working in multiagency teams to deliver holistic healthcare..$^{38} 5154$

Health professionals spoke of some difficulties referring refugees and asylum seekers to other health or social services. ${ }^{17} 1839405055$ Some, services were not set up to meet their needs, ${ }^{174}$ others would not receive referrals because they were operating at full capacity ${ }^{18} 39$ and sometimes services were simply not present. ${ }^{18} 55$ These challenges could be accentuated when health professionals found it difficult to navigate complex healthcare systems themselves. ${ }^{43}$

\section{Organisation}

Some articles highlighted flexibility in primary healthcare systems as beneficial for practice among refugees and asylum seekers. ${ }^{40} 41434950$ This allowed for innovative approaches to optimise service delivery ${ }^{40} 43$ such as relocating services to more accessible places ${ }^{1840} 4250$ and adaptation of working patterns to better suit service users' needs. ${ }^{4350}$

Provision of specialised services for refugees and asylum seekers was supported across some studies, ${ }^{3740} 4347$ including initial health assessment services ${ }^{47}$ specialist teams $^{40} 47$ and specialist centres. ${ }^{37} 47$ However, it was emphasised that these should integrate well into mainstream healthcare services. ${ }^{3740}$

\section{Resourcing and capacity}

Longer, more frequent appointments and utilisation of interpreters led to additional costs being incurred, ${ }^{18193743474951}$ which some felt was not taken into account in health system financing models. ${ }^{43} 4749$ Some participants did not think that they could deliver adequate care as a result of funding shortages, ${ }^{37}{ }^{55}$ with one study citing an example where interpreters were not able to be used because of lack of finance. ${ }^{55}$

Shortages in workforces were reported in some articles, ${ }^{46} 4749$ putting additional workload and stress onto health professionals. ${ }^{46}{ }^{49}$ Reported consequences of this were closures of services to new patients ${ }^{47} 49$ and health professionals leaving their posts, further exacerbating the problem. ${ }^{49}$ Interpreter shortages were also mentioned as a difficulty ${ }^{464956}$ along with inflexibility of their service operations. $^{374255}$

\section{Asylum and resettlement}

Further challenges were associated with the immigration status of, and legislative policy towards, refugees and asylum seekers. ${ }^{18} 19373940464759$ In some instances, health professionals were hindered in meeting health needs due to policy restrictions. ${ }^{40}$ Difficulties understanding the frequently changing policies towards, and entitlements for, refugees and asylum seekers were reported ${ }^{39} 40$ and uncertainty was expressed about healthcare pathways for this group on arrival in the host country. ${ }^{47}$ Some health professionals described conflicts in their professional duty to act as an advocate for their patients while requirements were placed on them to conduct assessments used to inform the asylum process. ${ }^{19} 46$ Another concern raised was a perception that service users were abusing the health and welfare systems, ${ }^{18} 374059$ such as feigning symptoms of post-traumatic stress disorder to further their asylum claims ${ }^{37}$ or illegal benefit claims. ${ }^{18}$ 


\section{DISCUSSION}

Three analytical constructs containing 11 descriptive themes were interpreted in the thematic synthesis. Challenges and facilitators were located within the healthcare encounter (trusting relationships; communication; cultural understanding; health and social conditions; time), working within the healthcare system (training and guidance; professional support; connecting with other services; organisation; resourcing and capacity) and asylum and resettlement.

The growing research field of 'cultural competence' identifies components that can be incorporated into practice to enhance quality of care towards ethnic minority groups and reduce healthcare inequalities. ${ }^{62}{ }^{63}$ Betancourt $e t a b^{62}$ defined cultural competence in healthcare as 'the ability of systems to provide care to patients with diverse values, beliefs and behaviours, including tailoring delivery to meet patients' social, cultural and linguistic needs' ${ }^{62}$ This literature mirrors themes interpreted in the current review, including trusting relationships, communication and cultural understanding, as key components that may be optimised to improve healthcare and reduce inequalities. ${ }^{62} 63$

Trusting relationships are essential for effective healthcare delivery. ${ }^{64-66}$ Murray et $a l^{67}$ identified continuity of relationship, time, interpersonal skills and 'getting to know patients' as enhancers of trust between health professionals and patients. The current review likewise recognised these elements, and it can be argued that even greater attention to trust-building is needed for refugees and asylum seekers, a vulnerable and ethnically diverse group who may be apprehensive about engagement with healthcare systems. ${ }^{689}$

Communication between health professionals and patients is also regarded as essential. ${ }^{70}$ Language discordance may compromise the quality of healthcare, lessening detection of ill health and referral to further healthcare. ${ }^{71}$ Health professionals in the current review consistently thought language barriers hindered their work with refugees and asylum seekers. The main strategy used to overcome language barriers was communication through interpreters, as is recommended in the wider literature. ${ }^{73-75}$ However, concerns were raised about the quality and availability of interpreters. Generally, it is recommended that professional interpreters are used, as they have been trained in professional standards, medical terminology and ethical issues. ${ }^{75} \mathrm{Ad}$ hoc interpreters such as family or community members may be used pragmatically, although this may diminish the quality of interpretation and threaten patient confidentiality. ${ }^{74}$ Remote interpretation, such as telephone or video services have been developed to provide more efficient and timely services. ${ }^{76} 77$ The merits of such services have been debated ${ }^{7677}$ and conflicting opinions were likewise given in this review. A systematic review ${ }^{77}$ reported no significant difference in patient and provider satisfaction between remote and face-to-face interpreters, although subsequent primary studies have suggested a significant preference for in-person interpreters. ${ }^{76}$

Consistent with other research, ${ }^{6-8}{ }^{11-13}$ health professionals encountered challenges dealing with complex physical, psychological and social problems of refugees and asylum seekers and did not always feel prepared to meet their needs. They also reported challenges in cross-cultural care such as different understandings of health, healthcare and healthcare systems, which introduced complications.

Participants in this review saw opportunities for improving care by working together with other health services and civil society. Identifying these organisations and possible areas of collaboration such as information sharing, referral pathways and joint service delivery may benefit health providers, health professionals and service users.

The organisation and delivery of primary healthcare services to refugees and asylum seekers is a growing research area, with service models being developed that integrate specialised components with existing structures. $^{78} 79$ A model innovated in Australia established 'Beacon practices', which have expanded capacity for refugee care and may flexibly resource local services. ${ }^{79}$ Such integrated services provide specialised resources without isolating refugees and asylum seekers from general practice, which was a concern raised by some participants in this review.

Health professionals and health services operate within, and are influenced by, the wider healthcare policy environment. Decisions made at a political and health system levels invariably impact on frontline clinical practice in areas such as resourcing priorities, health professional roles and healthcare access. ${ }^{80}$ Health professionals in this review recognised associated challenges, particularly when healthcare pathways were unclear and changeable. This emphasises the need for policy makers to provide consistent, clear and up-to-date guidance on asylum and resettlement health policy for health professionals.

\section{Public health implications}

A central concern in public health is reduction of inequalities in health and healthcare. ${ }^{81}{ }^{82}$ WHO has established a commission on the social determinants of health to support countries and recommend actions that address inequalities in health. ${ }^{82}$ Healthcare inequalities exist when certain groups systematically receive lower quality care than the general population, resulting in poorer health outcomes. ${ }^{80} 83$ These inequalities have been widely observed in healthcare provision to ethnic minority groups across a broad range of health services ${ }^{80}$ and has been highlighted as an issue for refugees and asylum seekers in the UK. ${ }^{21}$ However, through knowledge translation, where evidence is moved into practice, challenges and facilitators identified in this review may be mapped onto components of healthcare interventions that may minimise such healthcare inequalities. ${ }^{84}$ 
Reduction in healthcare inequalities will likely require targeting healthcare resources towards disadvantaged groups. ${ }^{79}$ For example, health professionals in this review highlighted the need for additional resources such as interpreter services, training and professional support to improve quality of care for refugees and asylum seekers.

\section{Recommendations}

\section{Practice}

Health professionals should be sufficiently resourced to meet the complex needs of refugees and asylum seekers. This should include provision of appropriate training on areas of cultural competence, asylum policies and process and health conditions. It is recommended that specific clinical guidelines are developed for provision of care to refugees and asylum seekers, drawing on the best available evidence. Further professional support should be given to those working with patients who present with complex psychological and social difficulties. Relevant, up-to-date information should be made available to inform health professionals about the needs of current waves of refugees and asylum seekers and other available services for referral and collaboration. Health providers should ensure adequate time is allocated for appointments with refugees and asylum seekers allowing space for trust building, communication and cultural understanding. They should develop infrastructure to ensure that trained interpreters are provided in a timely manner for appointments. Where resources permit, trained interpreters should be available with face-to-face and remote options (eg, via phone), depending on patients' preferences.

Policy

Healthcare policy makers and commissioners should recognise the complex needs of refugees and asylum seekers, providing enhanced resources for quality and equitable service provision. Adequate human resourcing would allow health professionals to spend the necessary time to follow best practice. Integration of specialised components with existing general practice may facilitate care. Asylum and resettlement policy makers should seek to promote continuity of relationship with healthcare providers, limiting relocations.

\section{Research}

Primary qualitative research could explore other healthcare professionals' experiences of caring for refugees and asylum seekers. For example, no studies of pharmacists' experiences were identified in this review. Further systematic reviews could be conducted to investigate experiences of health professionals working with refugees and asylum seekers in other areas of the healthcare system. A systematic review of challenges and facilitators for mental health professionals providing services to refugees and asylum seekers could inform service delivery for this group and searches in for this current review identified primary studies that could be included.

The outputs from this review may be used to inform service models for refugees and asylum seekers.
Healthcare evaluations may be conducted to evaluate these models and identify areas that are able to improve quality of care.

\section{Strengths and limitations}

An extensive and systematic search that was carried out across four databases complemented by reference and citation searches and it is therefore unlikely that published studies would have been overlooked. The inclusion of only English language studies may have led to under-representation of health professionals working in non-English speaking countries leading to a greater applicability to healthcare policy and practice in Englishspeaking high-income countries. It is also possible that the database searches may not have identified studies where refugees and asylum seekers were referred to as 'migrants' or 'immigrants'; however, the additional handsearches conducted would likely have identified any further key studies relevant for this review.

In study selection, titles and abstracts were screened by one reviewer, giving potential for selection bias or for relevant studies to be missed. By involving a second reviewer at the full-text selection stage, the study team sought to minimise bias, and supplementary searches of reference lists and citations reduced the potential for missing key studies. A second reviewer in data extraction could have reduced possibility of transcription errors, and in the quality appraisal stage could have minimised potential for biased assessment. Ideally, the analysis process would also have involved multiple reviewers in coding and formation of descriptive and analytical themes, bringing a wider perspective to interpretation.

Participants in this review were limited to the core clinical professions of nurses, primary care doctors and midwives. Other professionals, that may be part of primary healthcare teams, such as mental health workers, counsellors, physiotherapists and other community workers, were not included, raising a question about the transferability to more diverse primary healthcare teams. Studies including other professional groups report similar themes to the present review; however, those including mental health professionals may have a greater emphasis on secondary stress experienced when working with traumatised patients. ${ }^{8586}$ A further consideration for transferability of these findings is the combining of data from the three clinical professions as they have different care practices, interaction with patients and support networks, giving the potential to introduce imprecision to the findings.

A strength of syntheses of qualitative research is that concepts are translated across studies, with common themes described that may be more transferable to other contexts and a greater ability to inform policy and practice. ${ }^{2687}$ This contrasts with primary qualitative studies that are tied to their context and transference of findings is treated with caution. ${ }^{2687} \mathrm{On}$ the other hand, a perceived limitation of thematic syntheses is that they introduce a greater degree of abstraction from original experiences, sacrificing thickness of data and details found within the 
primary studies. ${ }^{88}$ In this case, given that refugees are not a homogeneous group, it is perhaps acceptable to emphasise only the more generalised themes that transcend the contexts of individual studies.

\section{CONCLUSIONS}

Many people continue to be displaced due to conflict and persecution, seeking sanctuary in high-income countries. Health professionals that provide primary healthcare for refugees and asylum seekers experience a range of challenges and facillitators; within the healthcare encounter, the environment of the healthcare system, and in the broader context of asylum and resettlement policy and process. The challenges and facilitators identified in this review may inform practices and policies that improve the quality of healthcare and minimise healthcare inequalities for refugees and asylum seekers.

Acknowledgements The authors acknowledge the contribution of Madeleine Flawn who was a second reviewer in study selection stage of the review.

Contributors LR conceived the study and was responsible for the study design, search strategy, data extraction, quality assessment and data analysis. LLJ provided methodological advice. LR drafted the manuscript which was revised with input from LLJ and SD. All authors approved the final version for publication.

Competing interests None declared.

Provenance and peer review Not commissioned; externally peer reviewed. Data sharing statement No additional data are available.

Open Access This is an Open Access article distributed in accordance with the terms of the Creative Commons Attribution (CC BY 4.0) license, which permits others to distribute, remix, adapt and build upon this work, for commercial use, provided the original work is properly cited. See: http://creativecommons.org/ licenses/by/4.0/

(C) Article author(s) (or their employer(s) unless otherwise stated in the text of the article) 2017. All rights reserved. No commercial use is permitted unless otherwise expressly granted.

\section{REFERENCES}

1. United Nations High Commissioner for Refugees. History of UNHCR. UNHCR, 2016. http://www.unhcr.org/pages/49c3646cbc.html (accessed Jan 2016).

2. United Nations High Commissioner for Refugees. Convention and Protocol Relating to the Status of Refugees. UNHCR, 2010. http:// www.unhcr.org/protection/basic/3b66c2aa10/convention-protocolrelating-status-refugees.html (accessed Jan 2016).

3. United Nations High Commissioner for Refugees. Global trends; Forced displacement in 2015. UNHCR, 2016. http://www.unhcr. org/statistics/unhcrstats/576408cd7/unhcr-global-trends-2015.html (accessed Aug 2016).

4. Aspinall P. Hidden needs. identifying key vulnerable groups in data collections: vulnerable Migrants, Gypsies and Travellers, Homeless People, and sex workers. Inclusion Health, 2014. https://www.gov.uk/ government/uploads/system/uploads/attachment_data/file/287805/ vulnerable_groups_data_collections.pdf (accessed Jan 2016).

5. Daniel M, Devine C, Gillespie R, et al. Helping new refugees integrate into the UK: baseline data analysis from the survey of New Refugees. UK Border Agency, 2010. https://www.gov.uk/government/uploads/ system/uploads/attachment_data/file/116069/horr36-report.pdf (accessed Jan 2016).

6. Clark RC, Mytton J. Estimating infectious disease in UK asylum seekers and refugees: a systematic review of prevalence studies. $J$ Public Health 2007;29:420-8.

7. Allotey P. The health of refugees: public health perspectives from crisis to settlement. Oxford: Oxford University Press, 2003.

8. Keygnaert I, Vettenburg N, Temmerman M. Hidden violence is silent rape: sexual and gender-based violence in refugees, asylum seekers and undocumented migrants in Belgium and the Netherlands. Cult Health Sex 2012;14:505-20.

9. Aptekman M, Rashid M, Wright V, et al. Unmet contraceptive needs among refugees. Can Fam Physician 2014;60:e613-9.

10. Amara AH, Aljunid SM. Noncommunicable diseases among urban refugees and asylum-seekers in developing countries: a neglected health care need. Global Health 2014;10:24.

11. Kalt A, Hossain M, Kiss L, et al. Asylum seekers, violence and health: a systematic review of research in high-income host countries. Am J Public Health 2013;103:e30-e42.

12. Miller KE, Worthington GJ, Muzurovic J, et al. Bosnian refugees and the stressors of exile: a narrative study. Am J Orthopsychiatry 2002;72:341-54.

13. Fazel M, Wheeler J, Danesh J. Prevalence of serious mental disorder in 7000 refugees resettled in western countries: a systematic review. Lancet 2005;365:1309-14.

14. Starfield B. Is primary care essential? Lancet 1994;344:1129-33.

15. World Health Organization. WHO Nursing and Midwifery Progress Report 2008-2012. Geneva: WHO, 2013. http://www.who.int/hrh/ nursing midwifery/NursingMidwiferyProgressReport.pdf?ua=1 (accessed Jan 2016).

16. Burke M. The perceived experiences of primary healthcare professionals in Ireland: interprofessional teamwork in practice. Doctoral dissertation 2016.

17. Jensen NK, Norredam M, Priebe S, et al. How do general practitioners experience providing care to refugees with mental health problems? A qualitative study from Denmark. BMC Fam Pract 2013;14:17.

18. Burchill J, Pevalin D. Barriers to effective practice for health visitors working with asylum seekers and refugees. Community Pract 2012;85:20-3.

19. Kurth E, Jaeger FN, Zemp E, et al. Reproductive health care for asylum-seeking women - a challenge for health professionals. BMC Public Health 2010;10:659.

20. O'Donnell CA, Burns N, Mair FS, et al. Reducing the health care burden for marginalised migrants: the potential role for primary care in Europe. Health Policy 2016;120:495-508.

21. Jones D, Gill PS. Refugees and primary care: tackling the inequalities. BMJ 1998;317:1444-6.

22. Suphanchaimat R, Kantamaturapoj K, Putthasri W, et al. Challenges in the provision of healthcare services for migrants: a systematic review through providers' lens. BMC Health Serv Res 2015;15:390.

23. Holloway I. Qualitative research in Nursing and Healthcare. Somerset: Wiley, 2013.

24. Pope C, van Royen P, Baker R. Qualitative methods in research on healthcare quality. Qual Saf Health Care 2002;11:148-52.

25. Harden A, Garcia J, Oliver S, et al. Applying systematic review methods to studies of people's views: an example from public health research. J Epidemiol Community Health 2004;58:794-800.

26. Finfgeld-Connett D. Generalizability and transferability of metasynthesis research findings. J Adv Nurs 2010;66:246-54.

27. Thomas J, Harden A. Methods for the thematic synthesis of qualitative research in systematic reviews. BMC Med Res Methodol 2008;8:45.

28. Barnett-Page E, Thomas J. Methods for the synthesis of qualitative research: a critical review. BMC Med Res Methodol 2009;9:1.

29. Tong A, Flemming K, Mclnnes E, et al. Enhancing transparency in reporting the synthesis of qualitative research: entreq. BMC Med Res Methodol 2012;12:1.

30. Cooke A, Smith D, Booth A. Beyond PICO: the SPIDER tool for qualitative evidence synthesis. Qual Health Res 2012;22:1435-43.

31. The World Bank. World Bank list of economies 2015 [Data File]. The World Bank, 2016. https://siteresources.worldbank.org/ DATASTATISTICS/Resources/CLASS.XLS (accessed Jan 2016).

32. The Migration Observatory. Who Counts as a Migrant? Definitions and their Consequences. The Migration Observatory, 2017. http:// www.migrationobservatory.ox.ac.uk/resources/briefings/who-countsas-a-migrant-definitions-and-their-consequences (accessed Mar 2017).

33. Hacker $\mathrm{K}$, Anies M, Folb BL, et al. Barriers to health care for undocumented immigrants: a literature review. Risk Manag Healthc Policy 2015;8:175-83.

34. Critical Appraisal Skills Programme. Qualitative Research Checklist. Critical Appraisal Skills Programme, 2014. http://media.wix.com/ugd /dded87_25658615020e427da194a325e7773d42.pdf (accessed Jan 2016).

35. Dixon-Woods $\mathrm{M}$, et al. How can systematic reviews incorporate qualitative research? A critical perspective. Qualitative Research 2006;6:27-44.

36. Passey ME, Longman JM, Robinson J, et al. Smoke-free homes: what are the barriers, motivators and enablers? A 
qualitative systematic review and thematic synthesis. BMJ Open 2016;6:e010260.

37. Begg H, Gill PS. Views of general practitioners towards refugees and asylum seekers: an interview study. Diversity Health Soc Care 2005;2:299-305.

38. Bennett S, Scammell J. Midwives caring for asylum-seeking women: research findings. Pract Midwife 2014;17:9-12.

39. Burchill J. Safeguarding vulnerable families: work with refugees and asylum seekers. Community Pract 2011;84:23-6.

40. Burchill J, Pevalin DJ. Demonstrating cultural competence within health-visiting practice: working with refugee and asylum-seeking families. Divers Equal Health Care 2014;11:151-9.

41. Carolan M, Cassar L. Pregnancy care for African refugee women in Australia: attendance at antenatal appointments. Evid Based Midwifery 2007;5:54-8.

42. Drennan VM, Joseph J. Health visiting and refugee families: issues in professional practice. J Adv Nurs 2005;49:155-63.

43. Farley R, Askew D, Kay M. Caring for refugees in general practice: perspectives from the coalface. Aust J Prim Health 2014;20:85-91.

44. Feldmann CT, Bensing JM, de Ruiiter A. Worries are the mother of many diseases: general practitioners and refugees in the Netherlands on stress, being ill and prejudice. Patient Educ Couns 2007:65:369-80.

45. Furler J, Kokanovic R, Dowrick C, et al. Managing depression among ethnic communities: a qualitative study. Ann Fam Med 2010;8:231-6.

46. Griffiths R, Emrys E, Finney Lamb C, et al. Operation Safe Haven: the needs of nurses caring for refugees. Int J Nurs Pract 2003;9:183-90.

47. Johnson DR, Ziersch AM, Burgess T. I don't think general practice should be the front line: experiences of general practitioners working with refugees in South Australia. Aust New Zealand Health Policy 2008;5:20.

48. Kokanovic R, May C, Dowrick C, et al. Negotiations of distress between East Timorese and vietnamese refugees and their family doctors in Melbourne. Sociol Health IIIn 2010;32:511-27.

49. Lawrence J, Kearns R. Exploring the 'fit' between people and providers: refugee health needs and health care services in $\mathrm{Mt}$ Roskill, Auckland, New Zealand. Health Soc Care Community 2005;13:451-61.

50. Riggs E, Davis E, Gibbs L, et al. Accessing maternal and child health services in Melbourne, Australia: reflections from refugee families and service providers. BMC Health Serv Res 2012;12:117.

51. Samarasinghe K, Fridlund B, Arvidsson B. Primary health care nurses' promotion of involuntary migrant families' health. Int Nurs Rev 2010;57:224-31.

52. Suurmond J, Seeleman C, Rupp I, et al. Cultural competence among nurse practitioners working with asylum seekers. Nurse Educ Today 2010;30:821-6.

53. Suurmond J, Rupp I, Seeleman C, et al. The first contacts between healthcare providers and newly-arrived asylum seekers: a qualitative study about which issues need to be addressed. Public Health 2013;127:668-73.

54. Tellep TL, Chim M, Murphy S, et al. Great suffering, great compassion: a transcultural opportunity for school nurses caring for Cambodian refugee children. J Transcult Nurs 2001;12:261-74.

55. Tobin CL, Murphy-Lawless J. Irish midwives' experiences of providing maternity care to non-Irish women seeking asylum. Int $J$ Womens Health 2014;6:159-69.

56. Twohig PL, Burge F, MacLachlan R. Pod people. Response of family physicians and family practice nurses to Kosovar refugees in Greenwood, NS. Can Fam Physician 2000;46:2220-5.

57. Yelland J, Riggs E, Wahidi S, et al. How do Australian maternity and early childhood health services identify and respond to the settlement experience and social context of refugee background families? BMC Pregnancy Childbirth 2014;14:348

58. Yelland J, Riggs E, Szwarc J, et al. Compromised communication: a qualitative study exploring Afghan families and health professionals' experience of interpreting support in Australian maternity care. BMJ Qual Saf 2016;25:e1

59. Crowley P. The mental health needs of adult asylum seekers in Newcastle upon Tyne. J Public Ment Health 2005;4:17-23.

60. Feldmann T. What do refugees and general practitioners say about medically unexplained physical symptoms? medical errors undermine trust in the GP. Huisarts Wet 2007;50:381-4.

61. Roemer MI. National health systems of the world. Oxford: Oxford University Press, 1993.

62. Betancourt J, Green A, Carillo J. Cultural competence in health care: emerging frameworks and practical approaches. The Commonwealth Fund, 2002. http://www.commonwealthfund.org/usr_doc/ betancourt_culturalcompetence_576.pdf (accessed Aug 2016).
63. Saha S, Beach MC, Cooper LA. Patient centeredness, cultural competence and healthcare quality. J Natl Med Assoc 2008;100:1275-85

64. Hall MA, Dugan E, Zheng B, et al. Trust in physicians and medical institutions: what is it, can it be measured, and does it matter? Milbank Q 2001;79:613-39.

65. Mainous AG, Baker R, Love MM, et al. Continuity of care and trust in one's physician: evidence from primary care in the United States and the United Kingdom. Fam Med 2001;33:22-7.

66. Mechanic $\mathrm{D}$. The functions and limitations of trust in the provision of medical care. J Health Polit Policy Law 1998;23:661-86.

67. Murray $B$, McCrone S. An integrative review of promoting trust in the patient-primary care provider relationship. J Adv Nurs 2015;71:3-23.

68. Duncan GF. Refugee Healthcare: towards healing Relationships. Canadian Social Science 2015;11:158-68.

69. Peterson P, Sackey D, Correa-Velez I, et al. Building trust: delivering health care to newly arrived refugees. 1: Mater UQ Centre for Primary Health Care Innovation, 2011. http://www.materonline.org. au/services/refugee-services/pdfs/building-trust_-delivering-healthcare-to-newl- (accessed Aug 2016).

70. Ong LM, de Haes JC, Hoos AM, et al. Doctor-patient communication: a review of the literature. Soc Sci Med 1995;40:903-18.

71. Bischoff A, Bovier PA, Rrustemi I, et al. Language barriers between nurses and asylum seekers: their impact on symptom reporting and referral. Soc Sci Med 2003;57:503-12.

72. Timmins CL. The impact of language barriers on the health care of Latinos in the United States: a review of the literature and guidelines for practice. J Midwifery Womens Health 2002;47:80-96.

73. Flores $\mathrm{G}$. The impact of medical interpreter services on the quality of health care: a systematic review. Med Care Res Rev 2005;62:255-99.

74. Karliner LS, Jacobs EA, Chen AH, et al. Do professional interpreters improve clinical care for patients with limited english proficiency? A systematic review of the literature. Health Serv Res 2007:42:727-54.

75. Mayo R, Parker VG, Sherrill WW, et al. Cutting Corners: provider Perceptions of Interpretation Services and factors related to use of an ad Hoc Interpreter. Hisp Health Care Int 2016;14:73-80.

76. Locatis C, Williamson D, Gould-Kabler C, et al. Comparing in-person, video, and telephonic medical interpretation. $J$ Gen Intern Med 2010;25:345-50.

77. Azarmina P, Wallace P. Remote interpretation in medical encounters: a systematic review. J Telemed Telecare 2005;11:140-5.

78. Feldman R. Primary health care for refugees and asylum seekers: a review of the literature and a framework for services. Public Health 2006;120:809-16.

79. Kay M, Jackson C, Nicholson C. Refugee health: a new model for delivering primary health care. Aust J Prim Health 2010;16:98-103.

80. Smedley BD, Stith AY, Nelson AR. Unequal treatment: confronting racial and ethnic disparities in health care. Washington, DC: National Academies Press, 2002.

81. Arcaya MC, Arcaya AL, Subramanian SV. [Inequalities in health: definitions, concepts, and theories]. Rev Panam Salud Publica 2015;38:27106-71.

82. World Health Organization. Closing the gap in a generation: health equity through action on the social determinants of health. Final Report of the Commission on Social Determinants of Health. Geneva: World Health Organization, 2008. http://www.who.int/social determinants/final report/csdh finalreport 2008.pdf (accessed Aug 2016).

83. Betancourt JR, Green AR, Carrillo JE, et al. Defining cultural competence: a practical framework for addressing racial/ ethnic disparities in health and health care. Public Health Rep 2003;118:293-302

84. Colquhoun H, Grimshaw J, Wensing M. Chapter 3.3b Mapping KT interventions to barriers and facilitators. In: Straus S, Tetroe J, Graham I, eds. Knowledge translation in health care: moving from evidence to practice. Oxford: Wiley-Blackwell, 2013:137-49.

85. Puvimanasinghe T, Denson LA, Augoustinos M, et al. Vicarious resilience and vicarious traumatisation: experiences of working with refugees and asylum seekers in South Australia. Transcult Psychiatry 2015;52:743-65.

86. Priebe S, Sandhu S, Dias S, et al. Good practice in health care for migrants: views and experiences of care professionals in 16 European countries. BMC Public Health 2011:11:11.

87. Levack WM. The role of qualitative metasynthesis in evidence-based physical therapy. Phys Ther Rev 2012;17:390-7.

88. Sandelowski M, Docherty S, Emden C. Qualitative metasynthesis: issues and techniques. Res Nurs Health 1997;20:365-71. 